\title{
DEMOGRAPHY AND SEX RATIO IN SOCIAL SPIDERS
}

\author{
STEven A. Frank ${ }^{1}$ \\ Department of Biology, University of Michigan, Ann Arbor, MI 48109-1048
}

\begin{abstract}
Spiders that live in large cooperative societies are scattered among several taxonomic groups. All quasisocial species appear to have female-biased sex ratios, while congeneric species with less advanced forms of sociality have $1: 1$ sex ratios. I present two sex-ratio models that examine the interaction between the genetic structure of populations and two aspects of colony demography - the changes as the colony grows larger in fecundity per female and in colony survivorship. In the first model, I assume that all members of the population produce the same sex ratio under all conditions (monomorphic strategy), while in the second model I assume that the sex ratio can be adjusted according to stage in the colony growth cycle (conditional strategy). The results of these models are consistent with the typically observed effects of within-sex competition among relatives; the sex ratio is biased toward the sex with less intense competition. In addition to these effects, a number of interesting interactions are revealed among relatedness, demography, and constraints of the sex-determining mechanism (monomorphic vs. conditional strategies). For example, when survivorship or fecundity increases as the colony grows larger, the predicted sex ratio becomes more female-biased. These demographic factors of changing viability and fecundity with changing colony size interact synergistically with relatedness, and the effect of this interaction on the sex ratio depends on the constraints of the sex-determining mechanism.

There are two related roles for the models presented here. First, these models provide some general predictions about how complex variables of demography and population structure interact in shaping the evolution of social spider sex ratios. Second, the development of the models illustrates a theoretical method that provides a simple description of complex interactions between demography and population structure, as well as an example of the synergistic interaction between relatedness and cooperation in the context of a particular life history.
\end{abstract}

Received August 8, 1986. Accepted June 9, 1987

Spiders that live in large cooperative societies occur in at least five genera scattered across four families (Buskirk, 1981). A single social unit typically contains hundreds or thousands of adult spiders living in a single large web, which is cooperatively built and maintained. Females do nearly all of the work, most females reproduce, and there is often shared responsibility for the tending of egg cases and rearing of young (Wickler, 1973; Lubin, 1982; Christenson, 1984). This advanced form of cooperation, which I refer to here as "social," is technically classified as quasisocial, and is more complex than forms of sociality observed in other spiders (Wilson, 1971 pp. 130-134; Buskirk, 1981). Social spiders provide an excellent opportunity for evaluating ideas about the evolution of social behavior (Darchen and Delage-Darchen, 1986), since these ideas have mostly been applied to Hymenoptera and their generality continues to be debated (Andersson, 1984).

\footnotetext{
1 Present address: Department of Zoology, University of California, Berkeley, CA 94720.
}

Life histories vary widely among the social spiders, and details of social structure are poorly known for most species. Recently, life histories and social biology were studied in Anelosimus eximius (Theridiidae) (Brach, 1975; Tapia and De Vries, 1980; Vollrath, 1982, 1986a, 1986b; Vollrath and Rohde-Arndt, 1983; Christenson, 1984; Avilés, 1986b; Smith, 1986), Achaeranea wau (Theridiidae) (Lubin, 1982, 1986; Lubin and Robinson, 1982; Lubin and Crozier, 1985), Agelena consociata (Agelenidae) (Darchen, 1978; Riechert, 1985; Riechert et al., 1986), and Mallos gregalis (Dictynidae) (Jackson, 1978a, 1978b; Tietjen, 1986).

One life-history trait that appears to be consistent among social spiders is a strikingly female-biased sex ratio (Darchen, 1967; Jackson, 1978b; Lubin and Robinson, 1982; Avilés, 1986a, 1986b, 1987; Vollrath, 1986a, 1986b). By contrast, nonsocial spiders typically have equal numbers of males and females. In two cases, subsocial species have $1: 1$ sex ratios, while congeneric social species have female-biased sex ratios (Anelosimus eximius [Vollrath, 1986a] and Achaearanea wau [Y. D. Lubin, un- 
publ.]). Since many aspects of social behavior affect the reproductive values of sons and daughters, the study of sex ratios provides a window onto the patterns of selection and constraints that have shaped the social structure of a population. I have isolated two factors that are likely to be important in shaping sex ratios of social spiders, and more generally, in the evolution of social structure and life history. The first factor is the genetic structure of the population, that is, the relatedness among individuals within the colonies. Avilés (1986a, $1986 b, 1987)$ and Vollrath $(1986 a)$ have recently addressed this aspect of sex-ratio evolution in social spiders. The second factor is the relationship between reproductive efficiency and colony size, where efficiency is measured as expected reproductive success per female and has both fecundity and colony-survivorship (viability) components.

The predicted qualitative trends in the sex ratio with respect to population structure and efficiency are simple. As relatedness within colonies increases, the sex ratio is expected to become more female-biased, since the levels of local mate competition and inbreeding increase (Vollrath, 1986a), or, alternatively, the strength of group selection increases (Avilés, 1986a, 1986b, 1987; see general reviews by Charnov [1982 pp. 67-92] and Frank [1986a]). As the rate of increase in reproductive efficiency increases with expanding colony size, the sex ratio is again expected to become more female-biased since the rate of colony expansion depends on the number of females produced in each generation. I present here formal models incorporating these two factors. The models reveal an interesting synergistic effect between population structure and colony efficiency, which in turn depends on constraints imposed by the sex-determining mechanism. The magnitude of the effects on sex ratio caused by these two demographic characters may also be evaluated. Beyond the implications for social spider evolution, the techniques employed in developing the models illustrate a general approach for studying the complex interactions between demography and population structure.

\section{General Assumptions}

The two models presented below are based on the following assumptions and definitions. i) A colony is founded by either a single spider or a group of spiders that is small in number relative to mature colony size. ii) Mating occurs within discrete generations. iii) The founding group produces offspring (referred to as generation 1), and the sex ratio (proportion of males) produced by this founding generation is $x_{0}$. These firstgeneration offspring grow to adulthood and produce the second generation, with a sex ratio of $x_{1}$. In general the sex ratio produced by the $j$ th generation is $x_{j}$. iv) The reproductive efficiency of the colony, measured by expected reproductive success per female, may increase as the colony grows larger. The efficiency in the $j$ th generation is $h^{(j)}$, which describes either fecundity or colony survivorship (see below for details). v) When the population is in sex-ratio equilibrium, maximum efficiency is achieved by the adults of the gth generation, who produce the $(g+1)$ th generation with a sex ratio of $x_{g}$. vi) There is no immigration into established colonies. From generation 1 to $g$ there is no emigration. After generation $g$, females emigrate in groups of one or more individuals, so that the number of females staying home to reproduce is constant in each generation. Dispersing groups from different colonies may mix before settling to form a new colony. The founding groups are of constant size in any particular population. vii) Sex-ratio control is by the mothers, so that $x_{j}$ is the sex ratio by the $j$ th generation of mothers when producing the $(j+1)$ th generation.

These assumptions are rigid when compared with actual social spider biology. The conclusions of the models are, however, reasonably insensitive to realistic adjustments in the assumptions. The robustness of the models is evaluated in the Discussion.

\section{Methods}

The evolutionarily stable strategy (ESS) method will be used here (Maynard Smith and Price, 1973; Maynard Smith, 1982; Taylor, 1988). First, assume that the population (collection of colonies) is in sex-ratio equilibrium and that the equilibrium values 
for the $j$ th generation females are $\left\{x_{j}^{*}\right\}$. Next, assume that rare individuals produce a sex ratio $\left\{x_{j}^{*}+\epsilon_{j}\right\}$, where each $\epsilon_{j}$ is small. If we can find the set of equilibrium sex ratios $\left\{x_{j}{ }_{j}\right\}$ such that, for each $j$, the fitness associated with $\left\{x_{j}^{*}\right\}$ is greater than the fitness associated with $\left\{x_{j}^{*}+\epsilon_{j}\right\}$ for any small $\epsilon_{j}$, we have found the ESS, which is a local fitness maximum.

The first task is to decide how to measure fitness. The reasonable choice is a method that will measure relative contribution to the gene pool in future generations, following Fisher's (1958 pp. 27-30) general argument about reproductive value. The idea that present behavior affects contribution to the gene pool several generations into the future has also been discussed in the context of sex ratios, where the concept is referred to as relative contribution to the "asymptotic gene pool" (Oster et al., 1977; Benford, 1978; Taylor, 1988, unpubl.).

Contribution to the asymptotic gene pool can be measured by first developing a measure of expected number of descendants, where, by construction, each descendant has equal reproductive value. This measure of long-term reproductive success, sometimes called an "adaptive function," can then be used in the Price $(1970,1972)$ equation, which translates reproductive success and patterns of interactions among relatives into a measure of genetic success (Grafen, 1985; Wade, 1985). A measure of long-term reproductive success under the spider model is obtained by assuming i) that an individual's number of descendants is proportional to the total number of dispersing groups produced by its colony, weighted by the individual's relative contribution to these dispersing groups and ii) that each of these descendants has equal reproductive value. This technique will be used to construct adaptive functions. Further arguments concerning this and other fitness measures used below are delayed until the Discussion.

The second task is to choose a method for finding the equilibrium sex-ratio values $\left\{x_{j}^{*}\right\}$ under the ESS criteria discussed above. The values $\left\{x_{j}^{*}\right\}$ are an ESS set if the current frequency, $q$, of rare deviants with sex ratio $\left\{x_{j}^{*}+\epsilon_{j}\right\}$ is greater than $q^{\prime}$, the rare deviants' projected contribution to the asymp- totic gene pool after one cycle of selection (P. D. Taylor [unpubl.] has presented a similar approach). In other words, we need to find $\left\{x^{*}{ }_{j}\right\}$ such that $\Delta q=q^{\prime}-q$ is negative for any set of small $\epsilon_{j}$. The Price equation is a tool for locating an ESS that is valid for any given population structure. The form of the Price equation most useful for locating an ESS is

$$
\begin{aligned}
\bar{w}^{(\infty)} \Delta q= & \operatorname{Cov}\left(w_{\mathrm{s}}^{(\infty)}, q_{\mathrm{s}}\right) \\
& +\sum_{\mathrm{s}} \alpha_{\mathrm{s}} R_{\mathrm{s}}\left(w_{\mathrm{si}}^{(\infty)}, q_{\mathrm{s} i}\right) V_{\mathrm{s}}\left(q_{\mathrm{s} i}\right),
\end{aligned}
$$

with the following definitions: $\bar{w}^{(\infty)}$ is proportional to the size of the asymptotic gene pool, as projected after one cycle of selection; an s-type colony has a frequency of deviant individuals $q_{\mathrm{s}}$, and has a projected contribution to the asymptotic gene pool in proportion to $w_{\mathrm{s}}^{(\infty)}$; the frequency of s-type colonies is $\alpha_{\mathrm{s}} ; q_{\mathrm{s} i}$ characterizes the phenotype of an individual (let $q_{\mathrm{sa}}=0$ if the individual produces the common equilibrium sex ratio, and $q_{\mathrm{sb}}=1$ if it produces a deviant sex ratio); $w_{s i}^{(\infty)}$ is proportional to the total reproductive value of an individual's descendants after one cycle of selection, which depends on the phenotypes of other members of the same s-type colony $\left(w_{\mathrm{sa}}^{(\infty)}\right.$ refers to individuals producing the common equilibrium sex ratio, and $w_{\mathrm{sb}}^{(\infty)}$ refers to individuals producing a deviant sex ratio); $R_{\mathrm{s}}$ is the regression coefficient of $w_{\mathrm{s} i}^{(\infty)}$ with respect to $q_{\mathrm{s} i}$, and $V_{\mathrm{s}}\left(q_{\mathrm{s} i}\right)$ is the variance in sex-ratio types (deviant or common) within an s-type group.

If we let $x^{*}{ }_{j}=x_{j \mathrm{a}}$ and $x^{*}{ }_{j}+\epsilon_{j}=x_{\mathrm{j}}$, then using a standard ESS technique, $x^{*}{ }_{j}$ can be found by setting to zero the derivative of $\bar{w}^{(\infty)} \Delta q$ with respect to $x_{j \mathrm{~b}}$, evaluating this expression at $x_{j \mathrm{~b}}=x_{j \mathrm{a}}=x^{*}$, and checking to insure that $x^{*}{ }_{j}$ is a local maximum.

\section{Monomorphic Strategy}

In this first model, I assume that there is a single sex ratio produced by all individuals, i.e., a monomorphic strategy. In the second model below, I allow individuals to adjust their sex ratios conditionally with respect to generation (size) of their colony in the growth and dispersal cycle. Notationally, a monomorphic strategy is described 
by the constraint $x^{*}{ }_{j}=x^{*}$ for all $j$, and for the search process, $x_{j \mathrm{a}}=x_{\mathrm{a}}, x_{j \mathrm{~b}}=x_{\mathrm{b}}$, and $\epsilon_{j}=\epsilon$ for all $j$. This condition may be viewed as a constraint imposed by the sex-determining mechanism, in that particular genetic mechanisms (e.g., haplodiploidy) appear to allow greater flexibility in conditional sex-ratio adjustment than do other genetic mechanisms (e.g., heterogametic sex chromosomes).

A solution $x^{*}$ will be obtained by finding expressions for $w_{\mathrm{s}}{ }^{(\infty)},{w_{\mathrm{sa}}}^{(\infty)}$ and $w_{\mathrm{sb}}{ }^{(\infty)}$, substituting into (1), and following the procedure outlined above. The value $w_{\mathrm{sb}}{ }^{(\infty)}$ is measured as the number of descendants of a b-type (deviant) foundress that emigrate from the colony during one cycle, where a cycle begins with the founding generation (generation 0) and ends with the death of a colony. The progeny produced by mothers of generation 0 through generation $g-1$ do not emigrate; from generation $g$ onward, a portion of the progeny stay at home to maintain a constant plateau-size colony, while the rest disperse. Cycle fitness of $a$ btype depends on the size of a plateau colony (the result of growth during generations 0 to $g-1$ ) and on the proportion of the colony expected to have descended from a btype foundress, multiplied by the expected number of dispersers per colony member (dispersal from generation $g$ to colony death) and by the proportion of dispersers that are expected to have descended from a b-type individual.

To develop a measure of cycle fitness, a measure is first needed for the effect of colony size on the reproductive efficiency of females living in an s-type colony during the $j$ th generation. Since the expected effect of colony size on reproductive efficiency is assumed to be the same for all females in the colony, it will often be convenient to refer to this measure as colony efficiency.

Two different interpretations of efficiency will be used, corresponding to fecundity, on the one hand, and colony survivorship, on the other. Since efficiency may increase with colony size, and since the colony size plateaus in the gth generation, let colony efficiency be one in $g$ and later generations. A simple way to describe colony efficiency in the $j$ th generation is as a function of the ratio of colony size in the $j$ th generation to final plateau size in the $g$ th and later generations. Define the colony efficiency of an s-type colony in the $j$ th generation as

$$
h_{\mathrm{s}}^{(j)}=\left[\frac{F_{\mathrm{s}}^{(j)}}{F^{(g)}}\right]^{\vartheta} \quad j \leq g-1,
$$

where $F_{\mathrm{s}}^{(j)}$ is the number of females in an s-type colony in the $j$ th generation, $F^{(g)}$ is the number of females in a plateau phase colony with no deviant individuals, and $\vartheta$ is a nonnegative parameter that determines the rate of increase in efficiency as the colony grows. The number of females is used since females do nearly all of the work in hunting, defending the colony, provisioning the young, and maintaining the web, and since the rate of colony growth depends on the number of reproductive females and their efficiency.

Next, a description is needed for the success, in each generation, of deviant b-types living within an s-type colony, denoted by $Z_{\mathrm{sb}}$, which is proportional to the number of grandprogeny of a deviant individual and, therefore, represents the fitness of b-types with respect to the total population. One commonly used measure is a modification of the Shaw-Mohler (Shaw and Mohler, 1953) equation that was first used by Hamilton (1967):

$$
Z_{\mathrm{sb}}=\frac{x_{\mathrm{b}}}{x_{\mathrm{s}}}\left(1-x_{\mathrm{s}}\right)+1-x_{\mathrm{b}}
$$

where $x_{\mathrm{s}}=p_{\mathrm{s}} x_{\mathrm{a}}+q_{\mathrm{s}} x_{\mathrm{b}}$, and $p_{\mathrm{s}}$ and $q_{\mathrm{s}}$ are the frequencies of a-types and b-types, respectively, in an s-type colony.

The total reproductive value, per cycle, of descendants by a b-type living in a s-type colony can now be written as

$$
\begin{aligned}
w_{\mathrm{sb}}{ }^{(\infty)} \propto & \left(\prod_{j=0}^{g-1} Z_{\mathrm{sb}} h_{\mathrm{s}}^{(j)}\right) \\
& \cdot\left(\sum_{i=g}^{\infty}\left[\frac{\delta Z_{\mathrm{sb}}}{2\left(1-x^{*}\right)}\right]^{i+1-g}\right) \\
= & \left(Z_{\mathrm{sb}}\right)^{g}\left(\frac{\delta Z_{\mathrm{sb}}}{2\left(1-x^{*}\right)-\delta Z_{\mathrm{sb}}}\right) \\
& \cdot \prod_{j=0}^{g-1} h_{\mathrm{s}}^{(j)},
\end{aligned}
$$

where $\delta$ is the probability, in each genera- 
tion, of $g$ and later generations successfully producing offspring, and $h_{\mathrm{s}}^{(1)}$ may be interpretated as either the probability of colony survival in generation $j$ or the relative fecundity of females. The first term in (2a) is the expected size of a plateau-phase colony, including a component for the probability that the colony survives to the dispersal phase if $h_{\mathrm{s}}^{(j)}$ is interpretated as a colonyviability measure, and the second term is the expected number of dispersing females produced per adult female in the colony. Each term is weighted by $Z_{\text {sb }}$, the success of b-types that live in an s-type colony, relative to the population as a whole.

There are two cases to consider. In the first, $h_{\mathrm{s}}{ }^{(1)}$ is a fecundity measure, and, in the second, it is a measure of the likelihood of colony survival. Since we are interested in $w_{\mathrm{sb}}{ }^{(\infty)}$ as a function of $x_{\mathrm{b}}$ or $x_{\mathrm{s}}$, it is useful to rewrite the product of the $h_{\mathrm{s}}^{(j)}$ values as

$$
\begin{aligned}
\prod_{j=0}^{g-1} h_{\mathrm{s}}^{(j)} & =\prod_{j=0}^{g-1} h^{(j)} X_{\mathrm{s}}^{\left[(1+\vartheta)^{j}-1\right]} \\
& =\left(X_{\mathrm{s}}\right)^{\Gamma} \prod_{j=0}^{g-1} h^{(j)}
\end{aligned}
$$

for fecundity, with

$$
\Gamma=\left(\frac{(1+\vartheta)^{g}-1}{\vartheta}\right)-g
$$

and as

$$
\begin{aligned}
\prod_{j=0}^{g-1} h^{(j)} & =\prod_{j=0}^{g-1} h^{(j)}\left(X_{\mathrm{s}}\right)^{\vartheta j} \\
& =\left(X_{\mathrm{s}}\right)^{\mathrm{r}} \prod_{j=0}^{g-1} h^{(j)}
\end{aligned}
$$

for survival, with

$$
\Gamma=\frac{\vartheta g(g-1)}{2}
$$

where $X_{\mathrm{s}}=\left[\left(1-x_{\mathrm{s}}\right) /\left(1-x^{*}\right)\right]$, and $h^{(j)}$ is the efficiency of a colony with no deviant individuals.

To obtain a solution, expressions are needed for substitution into (1) for $\operatorname{Cov}\left(w_{\mathrm{s}}^{(\infty)}\right.$, $\left.q_{\mathrm{s}}\right)$ and $R_{\mathrm{s}}\left(w_{\mathrm{s} i}^{(\infty)}, q_{\mathrm{s} i}\right)$ in terms of $x_{\mathrm{a}}, x_{\mathrm{b}}$, and $x_{\mathrm{s}}$. The value for $w_{\mathrm{s}}^{\left({ }^{(\infty)}\right.}$ is obtained from $w_{\mathrm{sb}}{ }^{(\infty)}$ by replacing $x_{\mathrm{b}}$ with $x_{\mathrm{s}}$ in (2b), and

$$
\operatorname{Cov}\left(w_{\mathrm{s}}^{(\infty)}, q_{\mathrm{s}}\right)=\sum_{\mathrm{s}} \alpha_{\mathrm{s}} w_{\mathrm{s}}^{(\infty)}\left(q_{\mathrm{s}}-q\right) .
$$

$R_{\mathrm{s}}\left(w_{\mathrm{s} i}{ }^{(\infty)}, q_{\mathrm{s} i}\right)$ is

$$
R_{\mathrm{s}}\left(w_{\mathrm{s} i}^{(\infty)}, q_{\mathrm{s} i}\right)=w_{\mathrm{sb}}^{(\infty)}-w_{\mathrm{sa}}^{(\infty)},
$$

since $q_{\mathrm{sb}}=1$ and $q_{\mathrm{sa}}=0$, where $w_{\mathrm{sa}}{ }^{(\infty)}$ is obtained by replacing $b$ with $a$ in $(2 b)$. Substituting into (1) and setting to zero the derivative of $\bar{w}^{(\infty)} \Delta q$ with respect to $x_{\mathrm{b}}$, evaluated at $x_{\mathrm{a}}=x_{\mathrm{b}}=x^{*}$, yields

$$
-2(\Gamma+G) V_{\mathrm{a}}+G\left(\frac{1-2 x}{x}\right) V_{\mathrm{wg}}=0
$$

where $\Gamma$ was given in (3c) for a fecundity model, and in (3f) for a colony-survival model, $G=g+1 /(1-\delta)$ is proportional to the expected life span of the colony, $V_{\mathrm{a}}$ is the variance in $q_{\mathrm{s}}$ among colonies, and $V_{\mathrm{wg}}$ is the average variance in $q_{\mathrm{s} i}$ within colonies. Solving yields

$$
x^{*}=\frac{(1-\rho)}{2\left[1+\rho\left(\frac{\Gamma}{G}\right)\right]}
$$

where $\rho=V_{\mathrm{a}} / V_{\mathrm{t}}$ and $V_{\mathrm{t}}=V_{\mathrm{a}}+V_{\mathrm{wg}}$ is the total variance in $q_{s i}$ in the population. The form of $\rho$ suggests that it is some sort of genetic correlation measure, and, following the conjecture by Frank (1986a, 1986b), supported in a similar study by Taylor (1988), the proper interpretation of $\rho$ in this situation appears to be a pedigree or regression coefficient of relatedness of the controlling genotype (mothers in this case) to a male or female (depending on the context) chosen randomly from the progeny generation within the colony.

The interpretation of the ESS is easier when the sex ratio is written in ratio form (genetic value of sons): (genetic value of daughters) (Frank, 1986a, 1986b), yielding

$$
(1-\rho):(1+\rho)+2 \rho \frac{\Gamma}{G} \text {. }
$$

The term for sons and the first term for daughters is $(1-\rho):(1+\rho)$, which is the result for this sort of multigeneration model when there is no association between colony size and efficiency (Frank, 1986a). For a multigeneration model in which the local 
carrying capacity is reached before the generation in which dispersal occurs (fecundity declines with increasing colony size), the genetic value of daughters is $(1+\rho)$, minus a discount for competition among daughters and female relatives once the carrying capacity has been reached (Frank, 1986a). This discount for females is the product of $\rho$ and the intensity of competition among females and, with respect to the genetic value of daughters, represents a negative synergism between relatedness and competitive interactions among females over resources necessary for reproduction.

The form of the second term of the genetic value of daughters in $(6), 2 \rho(\Gamma / G)$, suggests that in the spider model there is a positive synergism between relatedness, $\rho$, and cooperative interactions among females in generating or conserving resources necessary for reproduction or in increasing the likelihood of colony survival. This positive synergism has simple interpretations. For the case of fecundity, when the colony is in its early stages of growth and is increasing in efficiency in each generation, making a daughter i) confers a benefit equally to all members of the colony during the current generation, since fecundity depends on the number of females and ii) confers a benefit to all future colony members, since the colony will grow faster because it is more fecund and will become fecund faster since fecundity depends on colony growth. For the case of colony survival, an extra daughter increases survivorship equally for all members of the colony in the current progeny cohort and enhances the survivorship of future generations by increasing the size of future cohorts, but this case lacks the positive feedback between growth and efficiency that occurs in the fecundity case. The magnitude of the effect $(\Gamma)$ for fecundity [Eq. (3c)] is therefore greater than or equal to the magnitude of the effect for colony survival [Eq. (3f)]. In each, the increase in efficiency is the cooperative effect represented by $2 \Gamma / G$, and the genetic value of this cooperative effect depends on $\rho$, the mother's relatedness to females with whom her daughters interact.

It is useful to dissect the cooperation term, $2 \Gamma / G$, and gain some insights into what the model tells us about demography and sex ratio. $G=g+1 /(1-\delta)$ is the expected lifespan of a colony with nonzero cycle fitness, i.e., one that has successfully produced a $(g+1)$ th generation, which includes the first dispersing females. For the case in which efficiency has a fecundity interpretation, $\Gamma$, given in (3c), describes the rate at which the size of the plateau-phase colony increases with an increasingly female-biased sex ratio, $X_{\mathrm{s}}$, that can be attributed to increased efficiency during the $g$ generations of growth. When efficiency is interpretated as the probability of colony survivorship, $\Gamma$ in (3f) is the relative likelihood of a colony successfully achieving the plateau-phase size and, therefore, of successfully producing females that disperse. Limiting cases are instructive and apply equally to fecundity and survival interpretations. As $\vartheta \rightarrow 0$, the increase in efficiency with increasing colony size goes to zero $(\Gamma \rightarrow 0)$, and the cooperative effect disappears. If $g=1$, then the number of progeny produced by the founding generation is the plateau size of the colony, there is no opportunity for cooperative effects, and again $\Gamma=0$. As $\delta$ approaches one, the expected lifespan of the colony, $G$, approaches infinity; therefore the relative number of generations in which there is an opportunity for cooperation by producing extra daughters (the first $g$ generations) approaches zero, and $\Gamma \rightarrow 0$. In general, cooperative effects increase when $g$ is large and $\delta$ is small, so that opportunity for cooperation is relatively frequent, and also when $\vartheta$ is large, so that the effects of cooperation on efficiency are large. The effects of these parameters on the sex ratio are illustrated in Figure 1, for the case in which $\Gamma$ is interpreted as a measure of increasing fecundity with colony size. The trends are the same when $\Gamma$ is a measure of colony survival, but the magnitude of female bias is slightly less. For example, when $g=4, \delta=0.5$ (the graph in the middle of the upper row) and $\rho=\vartheta=0.5$ (dot in the center of the graph), then $x^{*}=0.1875$ when efficiency is interpreted as fecundity and $x^{*}=0.20$ when efficiency is interpreted as colony survivorship.

\section{Conditional Strategy}

Suppose that instead of producing the same sex ratio in every generation, $x_{j}^{*}=x^{*}$ for all $j$, a female spider can adjust the sex 
$\delta=0.15$
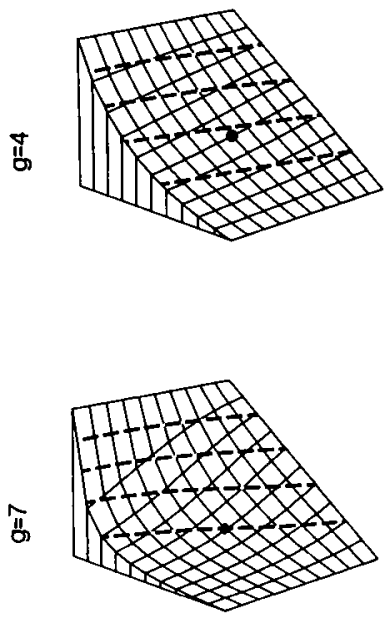

$\delta=0.5$
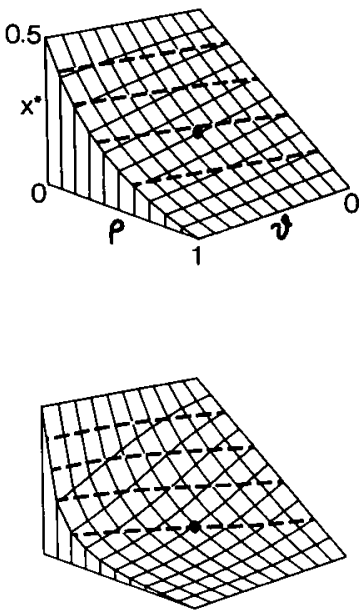

$\delta=0.85$
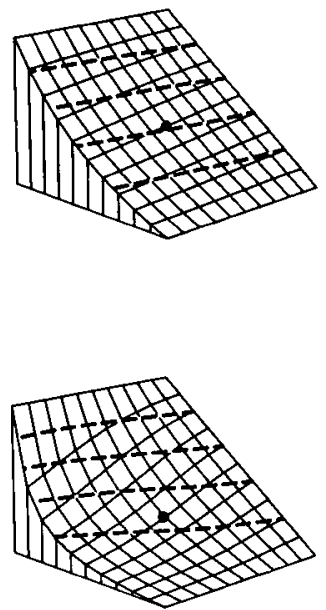

FrG. 1. Predicted sex ratios for the monomorphic-strategy model, where efficiency is interpreted as having an effect on fecundity. There are four parameters in the model: $\rho$ is the relatedness of the genotype that controls the sex (here assumed to be mothers) to a randomly chosen member of the colony from the progeny generation; $\vartheta$ determines the rate at which efficiency increases as colony size increases, where $\vartheta=0$ is no increase and $\vartheta=$ 1 is a linear increase; $g$ is the number of generations during which the colony grows without dispersing progeny; and $\delta$ is the probability, in each of the gth and later generations, that a colony survives and reproduces. The height of each graph, $x^{*}$, is the equilibrium sex ratio (males/total) predicted by the model, and the dashed lines mark sex-ratio contours at $0.1,0.2,0.3$, and 0.4 . The large dot at the center of each graph is at $\rho=\vartheta=0.5$.

ratio of her progeny conditional on her generation in the growth cycle of the colony or, equivalently, on the size of her colony compared with the size of a plateau-phase colony. Instead of $x_{\mathrm{a}}, x_{\mathrm{b}}$, and $x_{\mathrm{s}}$, we must now consider $\left\{x_{j \mathrm{a}}\right\},\left\{x_{j \mathrm{~b}}\right\}$ and $\left\{x_{j \mathrm{~s}}\right\}$. To develop a conditional model of this sort, a revised notion of cycle fitness is needed. In the monomorphic case above, $w_{\mathrm{sb}}{ }^{(\infty)}$ represents the projection of the total reproductive value of deviant b-types after one complete cycle of deviant sex-ratio behavior, where a cycle is from the founding generation until the colony dies. For a model in which sex ratio is conditional on generation, $j$, the proper measure of fitness is $w_{\mathrm{sb}}{ }^{(j \rightarrow \infty)}$, where $j \rightarrow \infty$ denotes the effect of deviant sex-ratio behavior, in the $j$ th generation, on the total reproductive value of descendants relative to the asymptotic gene pool. See the Discussion for a more complete argument about fitness measures.

The strategy for a solution $\left\{x_{j}^{*}\right\}$ is to assume, for each $j$, that $\mathbf{b}$-types produce a deviant sex ratio in the $j$ th generation and the normal equilibrium sex ratio in all other generations. The terms $w_{\mathrm{sb}}^{(j-\infty)}, w_{\mathrm{sa}}^{(j-\infty)}$, and $w_{\mathrm{s}}{ }^{(j-\infty)}$ are used to obtain the covariance and regression terms for (1), as in the monomorphic case, and then, for each $j, \bar{w}^{(\infty)} \Delta q$ is differentiated with respect to $x_{j \mathrm{~b}}$, evaluated at $x_{j \mathrm{~b}}=x_{j \mathrm{a}}=x^{*}$, and this derivative is set to zero. Since we will differentiate with respect to $x_{j \mathrm{~b}}$, we need only the part of $w_{\mathrm{sb}}{ }^{(j \rightarrow \infty)}$ that depends on $x_{\mathrm{j}}$. From (2b)

$$
w_{\mathrm{sb}}^{(j \rightarrow \infty)} \propto \begin{cases}Z_{j \mathrm{sb}} \prod_{k=j+1}^{g} h_{\mathrm{s}}^{(k)} & j<g \\ Z_{j \mathrm{sb}} & j \geq g\end{cases}
$$

where $Z_{j \mathrm{sb}}$ is the same as $Z_{\text {sb }}$ (see monomorphic case above), with $x_{\mathrm{a}}, x_{\mathrm{b}}$, and $x_{\mathrm{s}}$ replaced by $x_{j \mathrm{a}}, x_{j \mathrm{~b}}$ and $x_{j \mathrm{~s}}$. A more convenient form of $h_{\mathrm{s}}\left({ }^{(k)}\right.$ is needed for (7), as in (3),

$$
h_{\mathrm{s}}^{(k)}=h^{(k)} \prod_{d=0}^{k-1}\left(X_{d s}\right)^{\vartheta(1+\vartheta)^{k-d-1}}
$$

for fecundity, and

$$
h_{\mathrm{s}}{ }^{(k)}=h^{(k)}\left(X_{d \mathrm{~s}}\right)^{\vartheta}
$$

for colony survival where $X_{d \mathrm{~s}}=\left(1-x_{d \mathrm{~s}}\right) /$ 

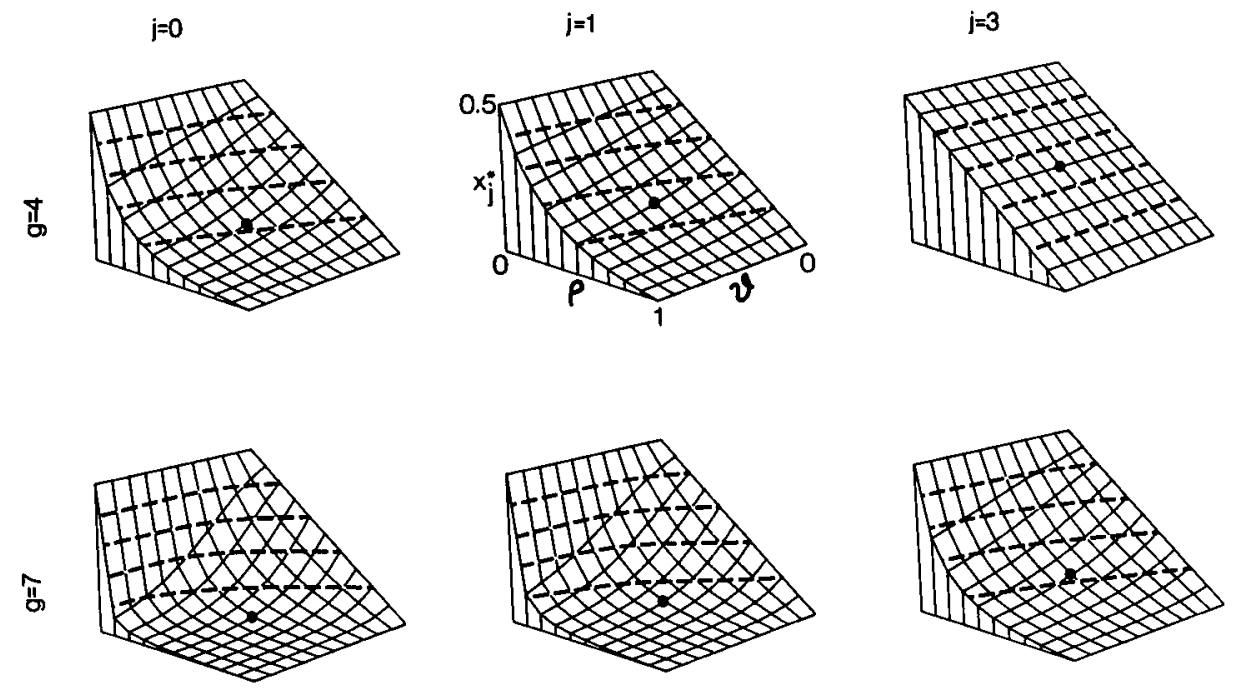

FIg. 2. Predicted sex ratios for the conditional-strategy model, where efficiency is interpreted as having an effect on fecundity. This figure is identical to Figure 1, except that the predicted sex ratio, $x^{*}{ }_{j}$ in the conditional model does not depend on $\delta$, but does depend on the generation, $j$, in the colony growth cycle.

( $1-x_{d}^{*}$ ), and $d$ is a subscript referring to generation. Following through yields

$$
x_{j}^{*}=\left\{\begin{array}{l}
\frac{1-\rho}{2\left(1+\rho \gamma_{j}\right)} \quad j<g-1 \\
\frac{(1-\rho)}{2} \quad j \geq g-1
\end{array}\right.
$$

with

$\gamma_{j}= \begin{cases}(1+\vartheta)^{g-1-j}-1 & j<g-1 \\ 0 & j \geq g-1\end{cases}$

for fecundity, and with

$$
\gamma_{j}= \begin{cases}(g-1-j) \vartheta & j<g-1 \\ 0 & j \geq g-1\end{cases}
$$

for colony survival. Equation (9a) in the ratio form of genetic value of sons to genetic value of daughters is

$$
(1-\rho):(1+\rho)+2 \rho \gamma_{j} .
$$

The relationship between the monomorphic and conditional solutions is simple and instructive, and it applies equally to fecundity- or survivorship-interpretations of efficiency. For colonies that successfully disperse individuals,

$$
E\left(\gamma_{j}\right)=\frac{1}{G} \sum_{j=0}^{\infty} \gamma_{j}=\frac{\Gamma}{G} .
$$

In words, $\Gamma / G$ is the average cooperative effect (on either fecundity or survival) of the conditional strategy over all generations, and this average is equivalent to the cooperative effect in the monomorphic case, shown in (6). When the spiders can adjust their sex ratio conditionally, the cooperative effect is strongest in the early generations and disappears once the colony reaches its plateau-phase size. The sex-ratio effects for the fecundity interpretation of efficiency are illustrated in Figure 2, for the same parameter values as Figure 1, except that $j$ now determines the conditional strategy and $\delta$ has no effect. The survivorship interpretation of efficiency yields the same trends, but with a slightly smaller effect on the magnitude of sex-ratio biases towards an excess of females. For example, the dot in the middle graph of the upper row is $x^{*}=0.154$, when efficiency is interpreted as fecundity, and would be $x^{*}=0.167$ for the same parameter values, if efficiency were interpreted as survivorship.

\section{DISCUSSION}

The theory presented in this paper has two functions. First, it has a place in formal 
sex-ratio theory and, more generally, in the theory of social evolution. Second, these models clarify patterns of selection that may be important for understanding social evolution in spiders and suggest a number of directions for future empirical and theoretical work.

\section{Formal Theory}

Methodology. - The use of Price's (1970, 1972) covariance method, or hierarchicalselection theory, for sex-ratio problems has been discussed in detail elsewhere (for the manner presented here, see Frank [1986a, $1986 b, 1987]$; for a different treatment, see Taylor [1988]). The essence of the method is to examine the effect of a current behavior on relative contribution to the gene pool at some time in the distant future (asymptotic gene pool [Oster et al., 1977; Benford, 1978; P. D. Taylor, unpubl.]) by combining Fisher's (1958 pp. 27-30) concept of reproductive value under complex demographic assumptions with Hamilton's (1964a, 1964b, 1972) concept of genetic value for interactions among relatives. Reproductive value is embodied in the adaptive function, which is a statement about the number of equally valuable descendants following one cycle of selection, when measured relative to the asymptotic gene pool. Reproductive value can then be translated into genetic value by use of the Price equation, which corrects for the effects of interactions among relatives. Here, I will discuss the multigeneration lifecycle measure of reproductive value-the adaptive function - that I used with the Price equation to derive the models presented in this paper.

The model assumed that the colony was founded by a small group of males and females or by already mated females. The individuals forming the founding group may be from different parent colonies; the only restriction is that there be a fixed number of females in the founding group. The colony grows for $g$ generations, without any immigration or emigration, and after $g$ generations the colony has reached its stable plateau-phase size. In the $(g+1)$ th and later generations most of the offspring disperse, and only enough remain at home to maintain the colony size. A measure of the number of direct descendants is needed, in which each descendant is weighted so that it has an equal reproductive value; in this context we need the effects of sex-ratio phenotypes on reproductive value after one cycle of selection. The definition of "one cycle of selection" must be considered with respect to the technique used to find the ESS.

One way to describe the method is to begin by assuming that there is a common allele and a rare allele at the locus that controls the sex ratio, and that both homozygotes and heterozygotes have the same sexratio phenotype. Suppose, however, that during one cycle of selection, the rare allele causes its bearers to produce a sex ratio that deviates slightly from the typical value. After this one cycle, both homozygotes and heterozygotes again produce the common sex ratio. Then, by using an adaptive function and the Price equation, one can project onto the asymptotic gene pool the change in the frequency of the rare allele. If the rare allele is less frequent in the asymptotic gene pool than before the deviant cycle, for any small deviation, then the common sex ratio must be an ESS (P. D. Taylor, unpubl.).

The definition of a "cycle" for the monomorphic case is straightforward. Recall that for a monomorphic strategy, the sex ratio must be the same in each generation of the growth- and plateau-phase of a colony, in spite of the possibility that selection may favor different phenotypes at different phases of colony growth. So, beginning with the founding generation and continuing on until the death of its colony, a deviant monomorphic phenotype will be subjected once to each selection regime, and thus this period is a single cycle of selection. Next, the results of one cycle of selection must be translated into an adaptive function-in other words, a statement about the numbers of descendants of equal reproductive value. The method used in this paper is to weight equally each group of potential foundresses dispersed by the colony and to weight the value of each group by the relative contribution of deviant types. The only difficult assumption implicit in this method is that the value of a group dispersing in a particular generation is exactly the same as the value of groups dispersing in later generations. For this particular sex-ratio model, the relative valuation of sons and daughters 
is not affected by a difference in the relative number of generations of future growth available to different sets of dispersers, since any advantage of extra generations is conferred equally to sons and daughters. Therefore, it is justified in this case to weight each dispersing group equally, independent of the generation in the plateau-phase in which they were born.

The method for constructing cycle fitness and asymptotic contribution differs for the conditional-strategy model. Since sex ratio is now conditional on generation number in the growth of the colony, each generation has to be treated separately. Suppose we are searching for the equilibrium sex ratio in the $j$ th generation, $x^{*}$. Once again, it is assumed that there is a common allele and a rare allele and that both homozygotes and heterozygotes typically produce the same sex ratio, although their common sex ratio now depends on generation in the colony. Only in the $j$ th generation of a single colonygrowth period is the slight deviation of the heterozygotes expressed. Therefore, one cycle of selection is simply the $j$ th generation of a particular colony-growth period, and is represented by $Z_{j \mathrm{sb}}$ in (7). The consequences of one cycle of selection with respect to numbers of equally valuable descendants are not as simple in this case. During the growth phase of a colony, extra daughters (but not extra sons) can increase the reproductive success of all progeny in the same and following generations by increasing the colony efficiency (either fecundity or survival); see Equation (7) for the case $j<g$, where cycle fitness, $Z_{j \mathrm{~b}}$, is weighted by an efficiency factor that depends on $x_{j}$. This weighting gives a proper measure of the total reproductive value of descendants as a function of deviant sex ratio in the $j$ th generation of one-colony growth period.

Fitness, Fecundity, and Colony Survival.-Colony efficiency is assumed to increase with increasing colony size in all models, at a rate described by the parameter $\vartheta$. For example, when $\vartheta \rightarrow 0$, there is no increase in efficiency with size, and when $\vartheta=1$, efficiency increases linearly with size. Efficiency in the models has two separate interpretations, each leading to similar qualitative results, which differ slightly in magnitude. In each case, genetic variability for the sex ratio among colonies leads to variability in efficiency, since the rate of colony growth depends on the sex ratio.

By one definition, efficiency describes the effect of colony size on the fecundity of females in the colony. As the colony grows larger, it may become more efficient in web maintenance, hunting, care for young, and so on, ultimately leading to more energy available for reproduction. Differences in efficiency among colonies lead to differences in fecundity and productivity among colonies and so may favor an increased femalebias in the sex ratio by the process of group selection, according to the amount of genetic differentiation among colonies (Colwell, 1981). I have presented the results of the models in an alternative and equivalent way (Frank, 1986a), by considering the interaction between increasing efficiency and relatedness in the local group, $\rho$. This allows causal mechanisms to be identified more clearly, as in (6) and (10) (see below).

By a second definition, efficiency describes the effect of colony size on the probability of colony survival (viability), which in turn affects individual survival, where survival increases with increasing colony size at a rate described by $\vartheta$. Differences in efficiency among colonies lead to differential rates of extinction and, therefore, may also favor an increased female bias by a process of group selection. Again, I used an alternative description. The production of more daughters (and fewer sons) by a female confers a benefit to colony members, related by $\rho$, because an extra daughter leads to greater colony growth and, therefore, a greater likelihood of survival.

Sex-Ratio Theory. - Past work on multigeneration sex-ratio models has been reviewed by Charnov (1982 pp. 72-73) and Frank (1986a). The main class of models assumes that each patch is colonized by a constant number of already mated females, the colony grows for a fixed number of generations, and then either the already mated females of the final generation disperse and begin the cycle again, or mating occurs randomly in the dispersal pool before females settle out and begin to colonize new patches 
to form the next generation. This model is called the haystack model (Bulmer and Taylor, 1980). In a previous paper (Frank $1986 a$ ), I studied a haystack model, with a monomorphic strategy, in which there is a fixed carrying capacity in each patch. I found that the earlier in the growth cycle that a colony reached its carrying capacity, the less female-biased its sex ratio would be (see also Wilson and Colwell [1981]). My interpretation of this result is that the density-independent equilibrium is favored early in the growth cycle, before the carrying capacity is reached, and that a 1:1 sex ratio is favored in those generations after the carrying capacity is reached. The monomorphic strategy is the result of a weighted average of the phenotypes favored by different selection regimes.

The models presented here are extensions of the haystack model. Instead of increasing competitive interactions with increasing density, an assumption of the spider model is that there is an increasing efficiency with increasing density, leading to increased fecundity or, alternatively, to increased colony survivorship. The conditional model discussed above shows that selection often favors different sex-ratio phenotypes in different generations, because of the changing effect with generation of current sex ratio on future efficiency. When extra females have a strong positive influence on future efficiency, then selection favors a more female-biased sex ratio. This effect is strongest in the earliest generation and declines steadily to zero when the colony reaches its plateau-phase size. The monomorphic strategy is simply the average of the conditional strategies, taken over all generations.

The conditional model clarifies the pattern of selection across generations. In addition, comparison between the conditional and monomorphic models provides a way to quantify the constraint of the sex-determining mechanism on the optimization of (additive) autosomal fitness. This type of measure would be useful when considering the joint evolution of sex ratio and sex-determining mechanism.

Social Evolution Theory. - An interesting result of these spider models is that the sex ratio is affected by an interaction between a cooperative-effects term and a genetic-relatedness term. The cooperative effects are the increased efficiency in future generations caused by extra females (and fewer males) produced by an individual in the $j$ th generation. The value of increased efficiency arising from extra females in the $j$ th generation is $\gamma_{j}$, and averaged over all generations is $\Gamma / G$. Since the optimal sex ratio can be viewed as the genetic value of sons relative to the genetic value of daughters, the genetic value of daughters is increased by $\rho \gamma_{j}$, the cooperative effects through increased efficiency conferred on all members of the colony, $\gamma_{j}$, weighted by the average relatedness, $\rho$, of the mothers (or unit controlling the sex ratio) to colony members. This synergism between cooperative effects and relatedness is of the general form presented by Queller (1985), and the synergistic interpretation arises naturally from the context of the spider model.

\section{Social Spider Biology}

The models have thus far been treated as formalisms, arising from a simple set of assumptions. I now examine these assumptions and their consequences in light of the biology of social spiders. First, I relate the predicted qualitative trends in the sex ratio and the assumptions of the model to the life history of social spiders. Second, I suggest ways to construct a more complete description of the traits that affect the sex ratio and social structure of spiders.

Both the monomorphic and conditional models make testable predictions about the relationship between sex ratio and patterns of colony growth and population structure. Many of these predictions have been mentioned above while developing and discussing the theory; however, it is useful to recast these predictions in a more applicable form. The predictions apply equally to both viability and fecundity interpretations of efficiency. The magnitudes of the effects tend to be slightly greater for the fecundity interpretation.

The first two predictions hold for both monomorphic and conditional strategies: i) the greater the genetic differentiation among colonies (relatedness within colonies, $\rho$ ), the 
more female-biased is the predicted sex ratio (Avilés, 1986b); ii) the greater the increase in colony efficiency with colony size, $\vartheta$, the more female-biased is the predicted sex ratio. The following predictions focus on differences between the two types of sexratio control: iii) if the sex ratio is monomorphic, then the greater the ratio of generations of colony growth, $g$, to generations in which the colony is at a constant plateau size, $G-g$, the more female-biased is the predicted sex ratio (Fig. 1); iv) if the sex ratio is conditional, the more generations remaining before the colony achieves its stable-plateau size, $g-j$, the more femalebiased is the predicted sex ratio (Fig. 2).

Although selection will usually favor conditional over monomorphic behavior, the advantage of conditional behavior may be negligible in some situations. A conditional strategy will be increasingly favored over a monomorphic strategy $v$ ) as the potential increase in efficiency caused by extra females, $\vartheta$, increases, vi) as the number of generations, $g$, in which the colony grows before reaching its plateau size increases, and vii) as the expected number of generations that the colony survives during its plateau phase, $G-g$, increases. The data presently available do not indicate that sex ratio varies with colony size in the social theridiids Achaearanea wau (Y. D. Lubin, unpubl.) and Anelosimus eximius (Avilés, $1986 b$; Vollrath, 1986b), although these data are not sufficient to rule out conditional behavior by these species. No data are available on whether the sex-determining mechanism might be sufficiently flexible to allow conditional behavior to evolve, but it is worth noting that conditional sex-ratio adjustment has been demonstrated in diploid aphids (Yamaguchi, 1985). See Vollrath (1986a) and Avilés (1986b) for a discussion of possible sex-determination mechanisms and their implications for social spider sex ratios.

These predictions suggest the sort of field data that is needed in order to gain a deeper appreciation of the patterns of selection and constraints that have shaped the sex ratios and social structures of spiders. However, these predictions are the logical results derived from a rather rigid set of assumptions about the life histories of social spiders (see
General Assumptions above). This set of assumptions was chosen both for making the mathematics tractable and for its robustness; in other words, the predictions are often insensitive to realistic departures from these assumptions. I now present some evidence and further arguments for my claim of robustness and point out where I feel extensions of the theory would be most useful.

In order to simplify the algebra, the models were based on a sharp change between colony growth with no dispersal early in the life cycle and dispersal with no further increase in colony size late in the life cycle. The switch between growth and dispersal in the models depends on generation number rather than colony size. Colony size and generation number are equivalent in the formal equilibrium models presented above, and therefore one may freely switch between size and generation-number interpretations of the models. It seems likely that colony size is the more important demographic variable in nature, with colonies perhaps switching from mainly growth to mainly dispersal above a certain size (see below), a trend for which these models are reasonably robust in their predictions.

The rate of increase in efficiency is parameterized by $\vartheta$, where $\vartheta=0$ implies that efficiency is independent of colony size. For example, if $\vartheta=0.5$ and the colony does not gain in efficiency after it has $N$ females, then efficiency at size $N / 2$ is $1 / \sqrt{2}=0.71$. A formal assumption of the model is that once a size of $N$ females is achieved, the colony disperses many individuals and grows no larger. The general trends predicted by the models require only that dispersal increases and that efficiency does not decline with increasing colony size (see Frank [1986a] for the case of declining fecundity). The general trends therefore hold even when $N$ is not the largest size obtained by the colony, but the size beyond which efficiency no longer changes. Formally, the model handles a wide range of efficiencies, so robustness is not a problem. The model could be extended so that efficiency is a general function of changing colony size-perhaps increasing while the colony is small and growing and then decreasing after it achieves some particular size. Most of the interesting predictions of 
the present formulation depend on efficiency increasing during the early phases of colony growth, so data on this point are of interest.

Long-term studies of colony growth and survivorship in social spiders are generally lacking. Small colonies, which are typically young, have lower survivorship than large colonies, which are typically older in the social species Anelosimus eximius (Vollrath, 1982), Agelena consociata (Riechert, 1985; Riechert et al., 1986), and Achaearanea wau (Lubin, unpubl.). Silk deposition per female declines considerably as the number of females per local group increases (Riechert et al., 1986; Tietjen, 1986). A ninefold decline was observed in laboratory studies of Mallos gregalis as the number of females increased over the range 1-20, with total web size and complexity also increasing (Tietjen, 1986). These data suggest that two components of efficiency increase with colony size: survivorship, and energy invested in web maintenance. However, competitive interactions among females of $A$. consociata may also increase with colony size, leading to lower fecundity (Riechert, 1985; Riechert et al., 1986). It is an open question whether the combined effects of changes in viability and fecundity with increasing colony size lead to an overall increase in expected reproductive success.

The models assume a rigid pattern of immigration, emigration, and colony growth (assumption vi). Colonies grow and do not disperse individuals during a fixed number of generations, $g$, and in later generations disperse a large proportion of individuals. In general, the qualitative conclusions of the model hold as long as there is a tendency for dispersal to increase with increasing colony size. These assumptions concerning growth and migration are based on the biology of Achaearanea wau, on one hand, and on an analogy with social insects, on the other. Colonies of $A$. wau grow for two or more generations without dispersal. Only large (old) colonies of $A$. wau produce dispersal swarms. The swarms generally constitute a large fraction of the colony population (Lubin and Robinson, 1982). Oster and Wilson (1978 p. 55) have shown, under a very wide range of assumptions, that social insect colonies are favored if they pro- duce only workers during the first several generations after founding, and then switch abruptly to making a large fraction of reproductives. The reasoning is similar to the spider model: social insect colony efficiency increases during the early generations and then eventually reaches a plateau-phase. Production of reproductives (dispersing individuals) generally begins when there is little potential for a further increase in efficiency. Oster and Wilson's (1978 p. 56) review of the available data suggests that this pattern of growth and reproduction is typical of social insects. This leads to another analogy with social insects: the extra females favored early in colony growth in the conditional spider model represent an increase in the worker force of the spider colony, since in all social spiders the females do the majority of the work in the web (Wickler, 1973; Brach, 1975; Tapia and De Vries, 1980; Lubin, 1982; Vollrath and Rohde-Arndt, 1983; Christenson, 1984). In this sense, a single female spider embodies both a worker and a reproductive.

While there is some preliminary evidence on colony growth and female movement, the situation for males is less clear and is a good focal point for empirical and theoretical studies. If the relatedness among colony members is high, then some dispersal may be favored, even though dispersal is likely to be very risky (Hamilton and May, 1977; Frank, 1986c). If males successfully emigrate into established colonies or founding groups, this would affect population structure, $\rho$, and would reduce the competition among male relatives. This would perhaps affect the genetic value of males, which would affect the predicted sex ratio. See Lubin (1986) for a discussion of mating competition among males and dispersal in Achaearanea wau. Electrophoretic studies of $A$. wau by Lubin and Crozier (1985) suggest that nearly all of the genetic variability in the study population is among geographically separated clusters of colonies. Similar results were obtained for Anelosimus eximius (Smith, 1986), and Agelena consociata (Riechert et al., 1986). Male migration over short distances may therefore have little effect on population structure.

In general, if migration between genetically differentiated groups is as rare as the 
present evidence suggests, the actual details of mixing will have little effect on the conclusions of the models. From a formal theoretical point of view, however, it is worth noting some uncertainties that may arise if migration is more common than presently believed. I have treated $\rho$ as a given parameter of the models, rather than a variable subject to selection. Previous experience suggests that this is often justified in an equilibrium analysis and that one may calculate $\rho$ based on the particular assumptions of migration and then substitute the result into the general model (compare Bulmer [1986] with Frank [1986b]). A difficulty may arise, however, since migration rates for spiders are likely to be under selection: changes in migration will affect patterns of interactions among male relatives, which influence the sex ratio. Formal robustness of these simple models can only be determined by a global, dynamic model that treats all of these factors simultaneously, and by further studies of male movement in natural populations.

The difficulties of simultaneously treating selection of migration rates and population structure, rather than treating population structure as a given parameter of the model, suggest that further theoretical work may reveal additional complexities important for understanding social spider evolution. The qualitative predictions are, however, reasonably insensitive to the patterns of migration, except perhaps for the presently unobserved case when males frequently migrate, and are robust when dispersal tends to increase with increasing colony size. The seven general predictions presented above are therefore useful and robust guidelines for future studies of sex ratio and social evolution in spiders.

\section{ACKNOWLEDGMENTS}

Y. Lubin introduced me to this problem, generously shared her knowledge of social spiders, and made many valuable criticisms of the presentation. L. Avilés and P. Smouse also gave me helpful advice on earlier drafts of the manuscript. Much of this work was completed while $Y$. Lubin and I were at Oxford. Our visit was generously arranged by W. D. Hamilton, and partly funded by the Royal Society. I was supported by NIH grants 1-T32-GM-07544-07 and RO1-GM32589 .

\section{Literature Cited}

ANDERSSON, M. 1984. The evolution of eusociality. Ann. Rev. Ecol. Syst. 15:165-189.

Avilés, L. 1986a. Sex ratio in the social spider Anelosimus eximius summary, p. 301. In W. G. Eberhard, Y. D. Lubin, and B. C. Robinson (eds.), Proceedings of the IX International Congress of Arachnology. Smithsonian Inst. Press, Wash., D.C.

- $1986 b$. Sex-ratio bias and possible group selection in the social spider Anelosimus eximius. Amer. Natur. 128:1-12.

- 1987. Female biased sex ratios in social spiders: A result of group selection? In Proceedings of the $\mathrm{X}$ International Congress of the International Union for the Study of Social Insects. Verlag-Dohann Beberny, Munich, W. Ger.

BENFORD, F. A. 1978. Fisher's theory of the sex ratio applied to the social Hymenoptera. J. Theoret. Biol. 72:701-727.

Brach, V. 1975. The biology of the social spider Anelosimus eximius (Araneae: Theridiidae). Bull. So. Calif. Acad. Sci. 74:37-41.

BULMER, M. G. 1986. Sex ratio theory in geographically structured populations. Heredity 56:69-73.

Bulmer, M. G., AND P. D. Taylor. 1980. Sex ratio under the haystack model. J. Theoret. Biol. 86:8389.

BUSKIRK, R. E. 1981. Sociality in the Arachnida, pp. 282-359. In H. R. Hermann (ed.), Social Insects. Academic Press, N.Y.

Charnov, E. L. 1982. The Theory of Sex Allocation. Princeton Univ. Press, Princeton, NJ.

Christenson, T. E. 1984. Behaviour of colonial and solitary spiders of the theridiid species Anelosimus eximius. Anim. Behav. 32:725-734.

ColWELl, R. K. 1981. Group selection is implicated in the evolution of female-biased sex ratios. Nature 290:401-404.

DARCHEN, R. 1967. Une nouvelle araignée sociale du Gabon, Agelena republicana Darchen (Araneide labidognathe). Biol. Gabon. 3:31-42.

-. 1978. Les essaimages de l'araignée sociale Agelena consociata Denis, dans la forêt gabonaise. C. R. Séances Soc. Biol. 287:1035-1037.

Darchen, R., AND B. Delage-Darchen. 1986. Societies of spiders compared to the societies of insects. J. Arachnol. 14:227-238.

FISHER, R. A. 1958. The Genetical Theory of Natural Selection, 2nd Ed. Dover, N.Y.

FrANK, S. A. 1986a. Hierarchical selection theory and sex ratios. I. General solutions for structured populations. Theoret. Popul. Biol. 29:312-342.

- 1986b. The genetic value of sons and daughters. Heredity $56: 351-354$.

- 1986c. Dispersal polymorphisms in subdivided populations. J. Theoret. Biol. 122:303-309.

- 1987. Variable sex ratio among colonies of ants. Behav. Ecol. Sociobiol. 20:195-201.

Grafen, A. 1985. A geometric view of relatedness. Oxford Surv, Evol. Biol. 2:28-89.

Hamilton, W. D. 1964a. The genetical theory of social behaviour. I. J. Theoret. Biol. 7:1-16. 
$1964 \mathrm{~b}$. The genetical theory of social behaviour. II. J. Theoret. Biol. 7:17-52.

. 1967. Extraordinary sex ratios. Science 156: $477-488$.

- 1972. Altruism and related phenomena, mainly in social insects. Ann. Rev. Ecol. Syst. 3: 193-232.

Hamilton, W. D., and R. M. May. 1977. Dispersal in a stable habitat. Nature 269:578-581.

JACKSON, R. R. 1978a. Comparative studies of Dictyna and Mallos (Araneae: Dictynidae). I. Social organization and web characteristics. Rev. Arachnol. 1:133-164.

- 1978b. Aggregations of Mallos and Dictyna (Araneae: Dictynidae): Population characteristics. Psyche 85:65-80.

LubiN, Y. D. 1982. Does the social spider, Achaearanea wau (Theridiidae), feed its young? Z. Tierpsychol. 60:127-134.

- 1986. Courtship and alternative mating tactics in a social spider. J. Arachnol. 14:239-257.

Lubin, Y. D. AND R. H. CrozIER. 1985. Electrophoretic evidence for population differentiation in a social spider. Insect. Soc. 32:297-304.

Lubin, Y. D., AND M. H. Robinson. 1982. Dispersal by swarming in a social spider. Science 216:319321.

Maynard SMITH, J. 1982. Evolution and the Theory of Games. Cambridge Univ. Press, Cambridge, U. K.

Maynard Smith, J., and G. R. Price. 1973. The logic of animal conflict. Nature 246:15-18.

Oster, G. F., I. Eshel, AND D. CoHEN. 1977. Workerqueen conflict and the evolution of social insects. Theoret. Popul. Biol. 12:49-85.

Oster, G. F., AND E. O. Wilson. 1978. Caste and Ecology in the Social Insects. Princeton Univ. Press, Princeton, NJ.

PrICE, G. R. 1970. Selection and covariance. Nature 227:520-521.

- 1972. Extension of covariance selection mathematics. Ann. Hum. Genet. 35:485-490.

QUELLER, D. C. 1985. Kinship, reciprocity and synergism in the evolution of social behaviour. Nature 318:366-367.

RIECHERT, S. E. 1985. Why do some spiders cooperate? Agelena consociata, a case study. Flor. Entomol. 68:105-116.
Riechert, S. E., R. RoelofF, AND A. C. ECHTERNACT. 1986. The ecology of the cooperative spider Agelena consociata in Equatorial Africa. J. Arachnol. 14:175-191.

Shaw, R. F., AND J. D. Mohler. 1953. The selective advantage of the sex ratio. Amer. Natur. 87:337342.

Smith, D. R. R. 1986. Population genetics of Anelosimus eximius (Areneae, Theridiidae). J. Arachnol. 14:201-217.

TAPIA, Y., AND T. DE VRIES. 1980. Tolerancia y cooperación en la araña social Anelosimus jucundus del bosque tropical Rio Palenque, Ecuador. Rev. Univ. Católica Quito, VII 27:51-74.

TAYLOR, P. D. 1988. Inclusive fitness models with two sexes. Theoret. Popul. Biol. In press.

TietJEN, W. J. 1986. Effects of colony size on web structure and behavior of the social spider Mallos gregalis (Areneae, Dictynidae). J. Arachnol. 14:145157.

Vollrath, F, 1982. Colony foundation in a social spider. Z. Tierpsychol. 60:313-324.

1986a. Eusociality and extraordinary sex ratio in the spider Anelosimus eximius (Araneae: Theridiidae). Behav. Ecol. Sociobiol. 18:283-287.

- $1986 b$. Environmental parameters, reproduction and the sex ratio of the social spider Anelosimus eximius. J. Arachnol. 14:267-281.

Vollrath, F., ANd D. Rohde-ARndt. 1983. Prey capture and feeding in the social spider Anelosimus eximius. Z. Tierpsychol. 61:334-340.

WADE, M. J. 1985. Soft selection, hard selection, kin selection and group selection. Amer. Natur. 125: 61-73.

WICKLER, W. 1973. Über Koloniengründung und soziale Bindung von Stegodyphus mimosarum Pavesi und anderen soziale Spinnen. Z. Tierpsychol. 32: 522-531.

Wilson, E. O. 1971. The Insect Societies. Harvard Univ. Press, Cambridge, MA.

Wilson, D. S., AND R. K. Colwell. 1981. Evolution of sex ratio in structured demes. Evolution 35:882897.

YAMAGUCHI, Y. 1985. Sex ratios of an aphid subject to local mate competition with variable maternal condition. Nature 318:360-362.

Corresponding Editor: R. H. Crozier 